\title{
ANN-based Structural Element Performance Model for Reliable Bridge Asset Management
}

\author{
J.B. Son \& J.H. Lee \& H. Guan \\ Griffith School of Engineering, Griffith University, Queensland, Australia \\ Y.C. Loo \& M. Blumenstein \\ Science, Environment, Engineering and Technology group Executive, Griffith University
}

\begin{abstract}
Bridge Management Systems (BMSs) have been developed to assist in the management of a large bridge network. Historical condition ratings obtained from bridge inspections are major resources for predicting future deteriorations via BMSs. Available historical condition ratings in most bridge agencies, however, are very limited, and thus posing a major barrier for predicting reliable future structural performance. To alleviate this problem, A Backward Prediction Model (BPM) technique has been developed to help generate missing historical condition ratings which is crucial for bridge deterioration models to be able to predict more accurate solutions. Nevertheless, there are still considerable limitations in the existing bridge deterioration models. In view of this, feasibility study of Time Delay Neural Network (TDNN) using BPMgenerated historical condition ratings is conducted as an alternative to existing bridge deterioration models. It is anticipated that the TDNN using BPM-generated data can lead to further improvement of the current BMS outcome.
\end{abstract}

\section{INTRODUCTION}

Bridges are one of the most important components in road transportation network. In order to ensure public safety and structural serviceability, wellestablished Maintenance, Repair and Rehabilitation (MR\&R) strategy is essential for optimal bridge asset management practice. To achieve this, Bridge Management Systems (BMSs) have been developed for effectively managing bridge networks. For a successful BMS, accurate prediction of future bridge condition is critical. Biennial bridge inspections containing condition ratings of bridge elements are the primary sources to obtain future prediction of bridge deteriorations in BMSs. However, insufficient bridge condition rating records in most bridge agencies are the major obstacle for predicting reliable structural performance.

The Artificial Neural Networks (ANNs) basedBackward Prediction Model (BPM) has been developed by Lee et al. (2008) to overcome the problem of insufficient condition ratings for reliable bridge deterioration prediction. This model can generate missing condition ratings through correlation between existing condition ratings and non-bridge factors which influence the variation of bridge condition ratings. It employs 21 non-bridge datasets (Lee et al. 2008) containing historical vehicle change, population change, climate change and precipitation change. In the present study, only 6 non-bridge fac- tors are employed to generate historical condition ratings, which was refined from the aforementioned 21 non-bridge factors used in the initial BPM development process. The 6 non-bridge factors contain passenger vehicle, truck, total number of vehicle, maximum temperature, local city population and state population growth. All the prediction errors obtained from the refined 6 non-bridge factors are found to be less than those obtained from the original 21 non-bridge factors.

Despite this improvement, the efficiency of newly generated condition ratings should be ascertained. To achieve this, the generated condition ratings are examined using specific prediction techniques commonly used in the bridge deterioration models. Based on the study of Morcous et al. (2002), the currently available bridge deterioration models can be summarised as deterministic, stochastic and Artificial Intelligence (AI). In both deterministic and stochastic models, missing historical data generated by the BPM technique can contribute to the improvement of prediction accuracy for future bridge conditions. Notwithstanding the above findings, several limitations of the above models are also worth noting. As for the deterministic models, these are: (1) prediction of the average condition of a bridge structure rather than condition ratings of individual elements; (2) neglecting the interaction between different bridge structure elements. For the stochastic models (i.e. Markov-based model), the limitations 
are: (1) it assumes condition state independency for simplicity, which means that future bridge condition depends only on the current condition but not on the historical condition, which is impractical; (2) only the overall condition ratings are presented for longterm prediction of bridges.

To overcome the abovementioned limitations in existing bridge deterioration models, feasibility study of Time Delay Neural Network (TDNN) using BPM-generated historical condition ratings has been conducted. It can make use of the benefits of the BPM-generated historical condition records which in turn would lead to effective bridge asset management.

\section{TIME-SERIES PREDICTION TECHNIQUES}

According to Bowerman and O'connell (1993), when prediction is used in decision-making, information related to events that have occurred in the past should be considered. Usually, historical data patterns are identified and are subsequently extended to future prediction. In most forecasting techniques, the prediction results therefore rely heavily on the assumption that the identified patterns from historical data will be extended to the future.

In particular, time series analysis has capabilities for prediction which is an important activity due to requirement of accurate forecasts in many decisionmaking processes.

\subsection{Auto-Regressive Integrated Moving Average (ARIMA)}

The Auto-Regressive Integrated Moving Average (ARIMA) model has been developed by Box and Jenkins (1970). This model is one of the most widely used techniques for reliable short-term predictions (Khashei et al. 2009). However, it has two significant limitations: (1) future value is assumed to be a linear function: and (2) large amount of historical dataset is required to obtain reliable predictions.

A recent research in relation to Artificial Neural Networks (ANNs) indicates that neural networkbased models provide more precise predictions than the traditional ARIMA-based models. This is because ARIMA models cannot fully capture the nonlinearity and chaotic behaviour of data due to functional linearity of the ARIMA (Kohzadi et al. 1996).

\subsection{Time-Delay Neural Networks (TDNNs)}

The neural-network based techniques have been applied to forecasting of nonlinear processes (Tan and Cauwenberghe 1999). One of such techniques, namely the TDNNs, originally designed for speech recognition (Waibel et al. 1989), has shown to produce reliable outcomes.
The TDNN model, developed by Waibel et al. (1989), has a multilayer feedforward network. The hidden and output neurons are replicated across time (Wang et al. 2007). It has a similar structure as the Multi-Layer Perceptron (MLP). Each layer in TDNN has one or more neurons which are connected for information processing. Figure 1 shows the structure of a typical TDNN. It describes that some neurons receive delayed input from other neurons within the same layer. For instance, the network receives a single input from the outside and the residual nodes in the input layer receive the input from the delayed neuron on the left-side by the one unit time interval (Zhong et al 2005).

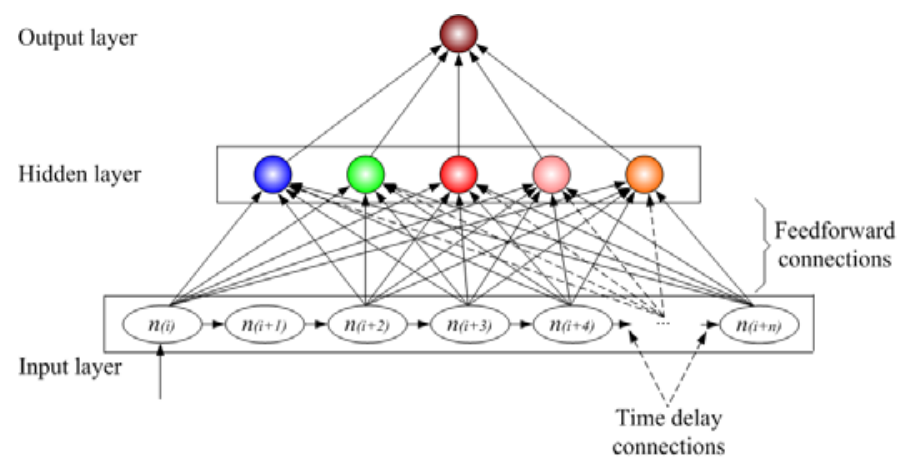

Figure 1. Structure of typical TDNN (Zhong et al 2005)

In this research, a sigmoid transfer function is selected because it produces a continuous value ranging from 0 to 1 . A neuron in a hidden layer $(n(j)$, $\left.n_{(j+1) \ldots} n_{(j+n)}\right)$ is linked to neurons $\left(n_{(i)}, n_{(i+1) \ldots} . . n_{(i+n)}\right)$ in the input layer. The connection from $n_{i}$ to $n_{j}$ has the weight $\mathrm{w}_{i j}$ that initially allocates a random value between 0 and 1 . The appropriate weights $\left(w_{i j}\right)$ are determined in the training stage of neural network.

Input to $n_{i}$ is calculated using the following equation:

$$
\text { Input }_{i}=\sum_{j=1}^{n} W_{i j} \times \text { output }_{j}
$$

Output from $n_{i}$ is obtained using a sigmoid transfer function as

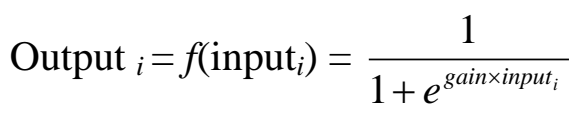

where the gain equals to a parameter that is utilised for adjustment of the slope in the sigmoid function. In most cases, -1 is a typical value used for the gain (Hecht-Nielsen 1990).

According to Hecht-Nielsen (1990), a neural network model has to be trained on the basis of a training set so that it adapts to the system it is trying to simulate. In the training stage, the weights of connections between neurons are adjusted to achieve the expected output. In particular, the back-propagation methodology is utilised to adjust the weights. Using this technique, errors from the output are fed back through the network to prevent error repetition. If the maximum training cycle or the predefined error thre- 
shold is reached, the training procedure stops and this neural network is then tested by using an independent dataset.

A feasibility study of TDNN technique is carried out in this study for long-term prediction of bridge condition ratings. This is based on TDNN's reliable outcome in dynamic analysis and its ability to capture non-linear characters of historical data (Zhu et al. 1998).

\section{FEASIBILITY STUDY OF BPM-BASED LONG-TERM PREDICTION USING TDNN}

Element-level historical condition ratings are essential resources in BMS software packages because they are used as input data in core modules such as condition rating and deterioration modules to analyse present and future conditions of bridges (Czepiel 1995). Accordingly, without sufficient condition rating datasets, the deterioration module is unable to provide reliable predictions of future bridge performance. As a result, an appropriate MR\&R plan cannot be provided through BMS analysis.

From bridge authorities' perspective, bridge condition ratings are still insufficient in most bridge agencies due to limited historical bridge inspection data. This is the major problem in the current BMSs. In addition, most available deterioration modellings have been designed to use only a few sets of historical condition rating records for long-term bridge deterioration predictions. These modelling techniques provide only an average condition of a bridge element. This indicates that such techniques ignore critical risks in bridge elements because small quantities of lower Condition States (CSs) of each bridge element are not considered.

To rectify the abovementioned problem, a deterioration model should provide future bridge conditions in a form of element-level condition ratings. A feasibility study of long-term prediction of bridge element deterioration is hereby conducted by using the BPM methodology in conjunction with the TDNN technique.

The procedure for future prediction of bridge condition ratings is presented in Figure 2. In Stage 1, the actual element-level bridge inspection records (years from $t_{1}$ to $t_{2}$ ) are correlated with relevant nonbridge factors to generate missing historical condition ratings (years from $t_{0}$ to $t_{1}$ ). The BPM methodology is used in this stage. Each year of the BPM outcome, i.e. generated condition ratings, contains 66 data which is the combined number of learning rates (lr: 0.0-0.5) and momentum coefficients (mc: $0.0-1.0$ ) in the neural network configurations. The number 66 also represents the total quantity of a given bridge element. The forward comparison method is used in the BPM methodology to validate the $\mathrm{BPM}$ results.
Stage 1 produces forward prediction (years from $t_{1}$ to $t_{2}$ ) using BPM outcomes (years from $t_{0}$ to $t_{1}$ ). The results of the forward predictions are then compared with the actual BMS condition ratings (years from $t_{1}$ to $t_{2}$ ). Subsequently, the generated historical condition ratings are ready for use in Stage 2 following validation of the BPM outcomes.

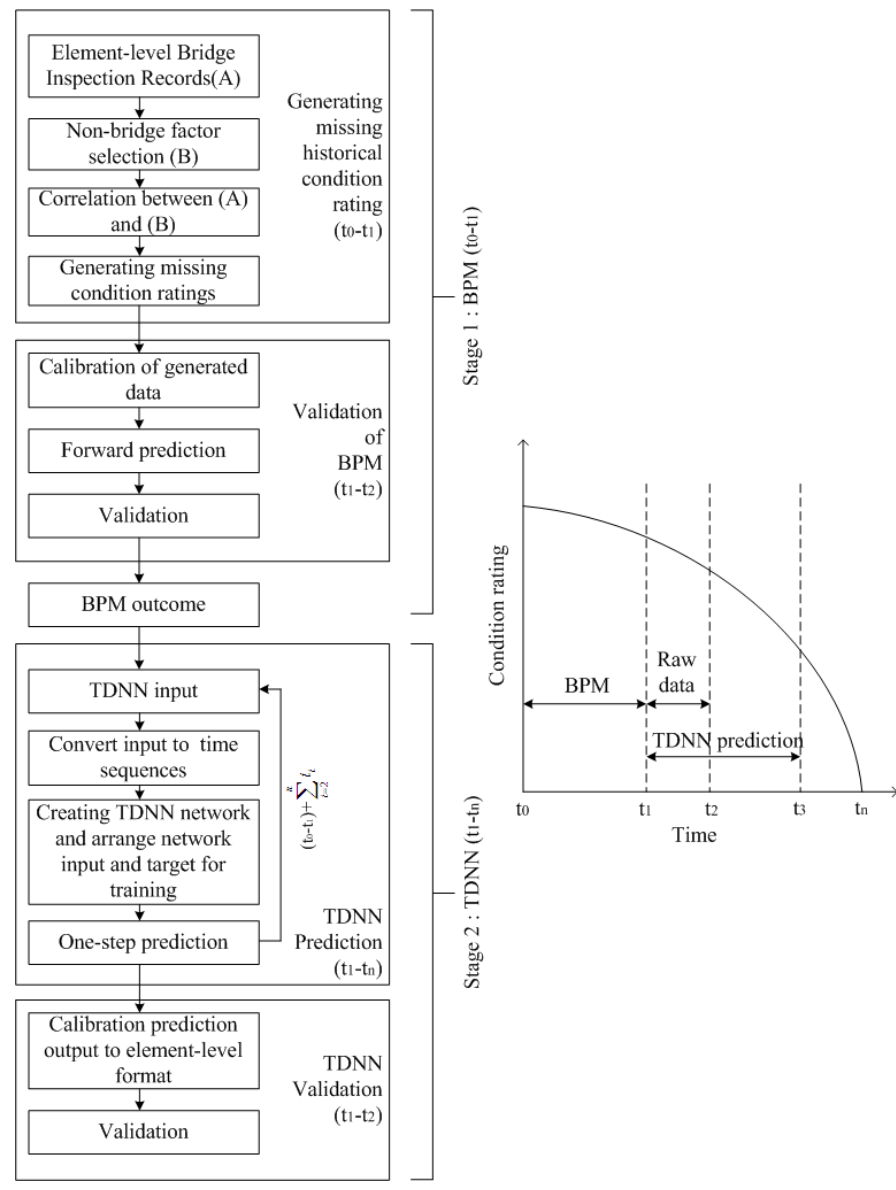

Figure 2. Procedure for prediction of future bridge condition ratings

In Stage 2, backward prediction results (from years $t_{0}$ to $t_{1}$ ) obtained from Stage 1 are used as TDNN inputs. These input data are converted to time sequences for time series prediction in the TDNN. The proposed TDNN provides only one-step ahead prediction at a time (one cycle), i.e. a 2-year interval in the actual time domain. The result of the first onestep-ahead prediction is added on to the original TDNN input (from years $t_{0}$ to $t_{1}$ ). This means that the amount of input for TDNN increases for the second cycle of the one-step-ahead prediction. To obtain a complete long-term prediction, iterations of the above-described process are required until the end of the life cycle $\left(t_{n}\right)$ of a given bridge element. The number of yearly prediction by TDNN is also 66 , which is in an identical form as the BPM outcomes obtained in Stage 1. The cross-validation is also necessary to measure prediction accuracy of TDNN outcomes. In this proposed study, the results of TDNN predictions (years from $t_{1}$ to $t_{2}$ ) are compared with the known (or "actual") BMS condition 
ratings (years from $t_{1}$ to $t_{2}$ ). All TDNN outcomes are considered satisfactory if the comparisons are within the error allowances. It should be noted that the final outcomes of the TDNN, upon calibration, are in the same form as the bridge element-level inspection. In summary, Stages 1 and 2 constitute the entire process of long-term deterioration prediction of one type of bridge elements.

The training algorithm used in TDNN is the backpropagation algorithm which is utilised in conjunction with a feed-forward multi-layer network. Backpropagation algorithm has shown to yield better results than traditional approximation methodologies in practical applications.

The log-sigmoid function is used in TDNN as a typical neuronal non-linear transfer function. The input layer has 14 BPM-generated historical condition ratings years from 1968 to 1994 which are used to predict unknown condition ratings from year 1996 onwards in the output layer. The specification for the inputs, outputs and functions of the TDNN are detailed in Table 1.

Table 1. Component of TDNN

\begin{tabular}{ll}
\hline Training algorithm & Back propagation algorithm \\
\hline Transfer function & Log-sigmoid function \\
\hline Inputs & $\begin{array}{l}\text { 14 BPM-generated historical condition } \\
\text { ratings (years from 1968 to 1994) }\end{array}$ \\
\hline Hidden layers & 2 (2 neurons) \\
\hline Output & $\begin{array}{l}\text { Bridge condition ratings } \\
\text { (1 output @ each year) }\end{array}$ \\
\hline
\end{tabular}

\subsection{Timeframe of proposed deterioration model}

The neural network based BPM has been developed recently (Lee et al. 2008) to overcome the shortcoming of missing historical condition ratings. This model generates unavailable historical condition ratings through the correlation between existing condition ratings and relevant non-bridge factors. The BPM-generated historical condition ratings are used as input for the proposed TDNN-based long-term deterioration model.

In this feasibility study, only available are five actual condition ratings (years 1996 - 2004 with a 2year increment) for both BPM and TDNN. Figure 3 describes the timeframe of the proposed deterioration model using the TDNN. The timeframe shows, for different years: (a) available condition ratings; (b) BPM inputs; (c) BPM results viz the generated historical condition ratings; (d) TDNN input; (e) TDNN prediction results; and (f) the cross-validation of the TDNN results.

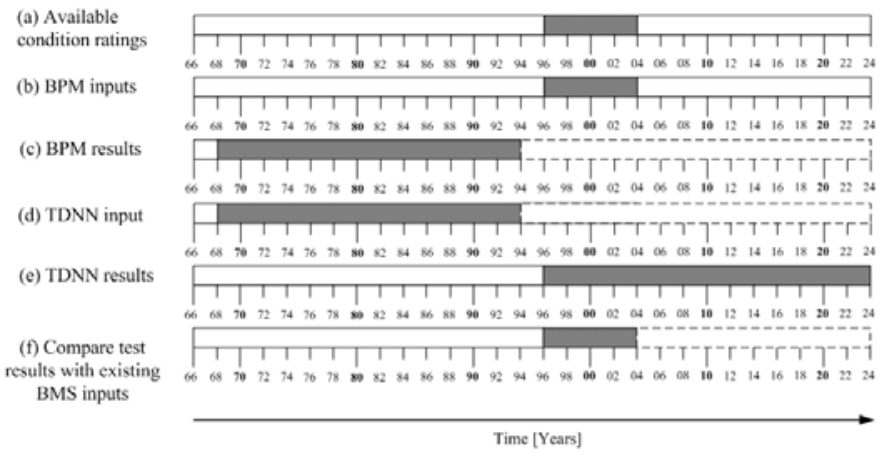

Figure 3. Timeframe of the proposed deterioration model (Element \#234 in Bridge \#0301xxxx1)

\subsection{Element-level prediction using TDNN}

As outlined in Figure 3, the actual element-level bridge inspection records (years from 1996 to 2004) are correlated with relevant non-bridge factors to generate missing historical condition ratings (years 1968 - 1994) by using the BPM methodology. The forward comparison method is used to validate the BPM results. It produces forward prediction (years 1996 - 2004) using BPM outcomes (years 1968 1994). The results of the forward predictions are then compared with the actual BMS condition ratings (years 1996 - 2004).

Secondly, the backward prediction results (years 1968 - 1994) obtained from the BPM methodology are used as TDNN inputs. These input data are converted to time sequences for time series prediction in the TDNN. The proposed TDNN provides only onestep ahead prediction at a time (one cycle). The result of the first one-step-ahead prediction (year 1996) is added on to the original TDNN input (years 1968 - 1994). Iterations of the above-described process are required for a given bridge element until the target year (year 2024). Cross-validation is also necessary to measure prediction accuracy of the TDNN outcomes. In this proposed study, the results of TDNN predictions (years 1996 - 2024) are compared with the known BMS condition ratings (years 1996 - 2004). All the TDNN outcomes are considered satisfactory if the comparison results are within the error allowances.

Figure 4 demonstrates 66 combinations of learning rates and momentum coefficients in BPM results (years 1966 - 1994) which are used to predict future bridge condition ratings (years 1996 - 2024) because the TDNN results cannot be determined by only one historical data pattern in an element-level prediction. The average quantity of each CSs on element \#234 (Reinforced Concrete Pier Cap) between 1996 and 2004 is about $80 \%, 16.2 \%$ and $3.8 \%$ of the total element in CS1, CS2 and CS3, respectively. Therefore, TDNN prediction using BPM-generated historical condition ratings is performed for three different CSs to consider proportion in each CSs.

The format of the TDNN prediction results are modified through simple post-calibration process to 
conform to the type of element level inspection. All the TDNN prediction results (years 1996 - 2024) are also summarised in Table 2.

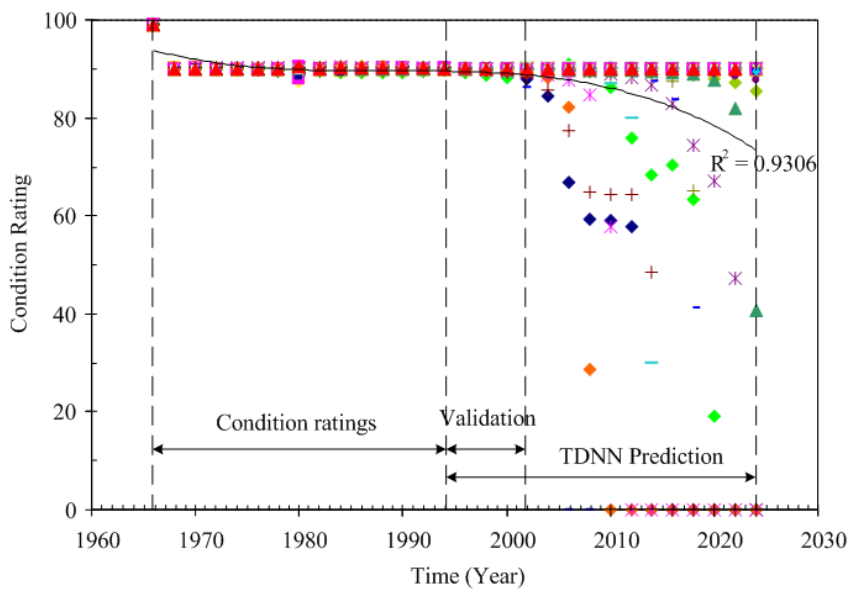

(a) About $80 \%$ of the total elements in CS1

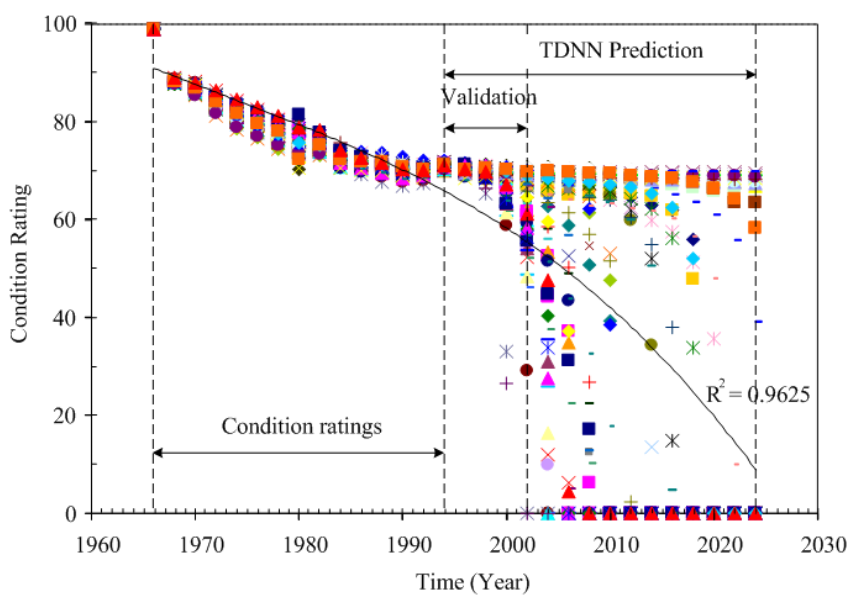

(b) About $16.2 \%$ of the total elements in CS2

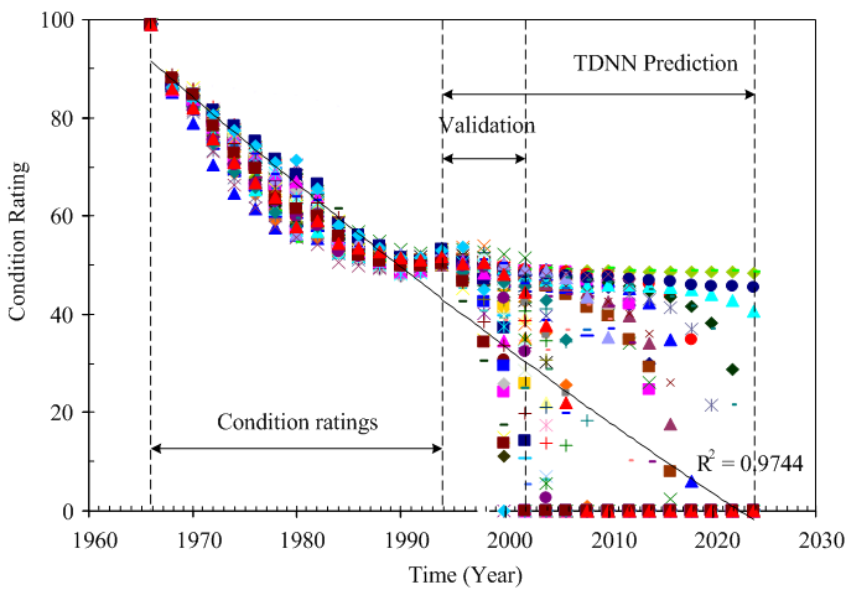

(c) About 3.8\% of the total elements in CS3

Figure 4. TDNN Prediction Results (Years 1996-2024)

The $\mathrm{R}^{2}$ values in each CS are statistical measurements representing the accuracy of approximation of the regression lines to the corresponding average point obtained from 66 element-level prediction results. Note that a third-order polynomial is used to present the regression lines shown in Figure 4.
Table 2. TDNN Prediction Results (Years 1996-2004) in element level inspection form

\begin{tabular}{|c|c|c|c|c|c|c|}
\hline Year & 1996 & 1998 & 2000 & 2002 & 2004 & 2006 \\
\hline CS1 & 280 & 280 & 280 & 280 & 276 & 266 \\
\hline CS2 & 57 & 54 & 54 & 42 & 34 & 39 \\
\hline CS3 & 13 & 10 & 10 & 19 & 15 & 11 \\
\hline CS4 & 0 & 4 & 4 & 4 & 8 & 7 \\
\hline CS5 & 0 & 2 & 2 & 5 & 18 & 28 \\
\hline Total & 350 & 350 & 350 & 350 & 350 & 350 \\
\hline Year & 2008 & 2010 & 2012 & 2014 & 2016 & 2018 \\
\hline CS1 & 263 & 259 & 252 & 252 & 252 & 238 \\
\hline CS2 & 33 & 30 & 39 & 25 & 25 & 28 \\
\hline CS3 & 11 & 14 & 7 & 7 & 4 & 11 \\
\hline CS4 & 7 & 3 & 0 & 7 & 0 & 0 \\
\hline CS5 & 36 & 44 & 53 & 60 & 70 & 74 \\
\hline Total & 350 & 350 & 350 & 350 & 350 & 350 \\
\hline Year & 2020 & 2022 & 2024 & & & \\
\hline CS1 & 238 & 238 & 235 & & & \\
\hline CS2 & 21 & 14 & 14 & & & \\
\hline CS3 & 2 & 7 & 7 & & & \\
\hline CS4 & 1 & 0 & 0 & & & \\
\hline CS5 & 89 & 91 & 95 & & & \\
\hline Total & 350 & 350 & 350 & & & \\
\hline
\end{tabular}

\section{MEASURING PREDICTION ACCURACY}

Feng et al. (2006) used cross-validation methodology, sometimes called rotation estimation (Geisser 1993), to validate regression and neural network predictions. Cross-validation involves partitioning existing data into supplementary dataset, performing the analysis on one dataset, and validating the analysis on the other dataset (Geisser 1993).

In this feasibility study, the cross-validation method is used to measure prediction accuracy of the proposed TDNN based model. The same years (1996-2004) of TDNN prediction results and the known BMS condition rating records are compared. In order to conduct the cross-validation, the results of TDNN predictions require calibrations, which is an identical process used in the BPM methodology. The results are in the element-level inspection form as shown in Table 2 . The cross-validation results are shown in Figure 5. The yearly average prediction errors are less than $\pm 10 \%$, i.e. (year 1996: $0.8 \%$, year 1998: $1.16 \%$, year 2000: $1.16 \%$, year 2002: $2.64 \%$ and year 2004: 4.22\%), which are considered acceptable. Therefore, predictions using TDNN based on BPM-generated historical condition ratings are regarded as reliable outcomes. 


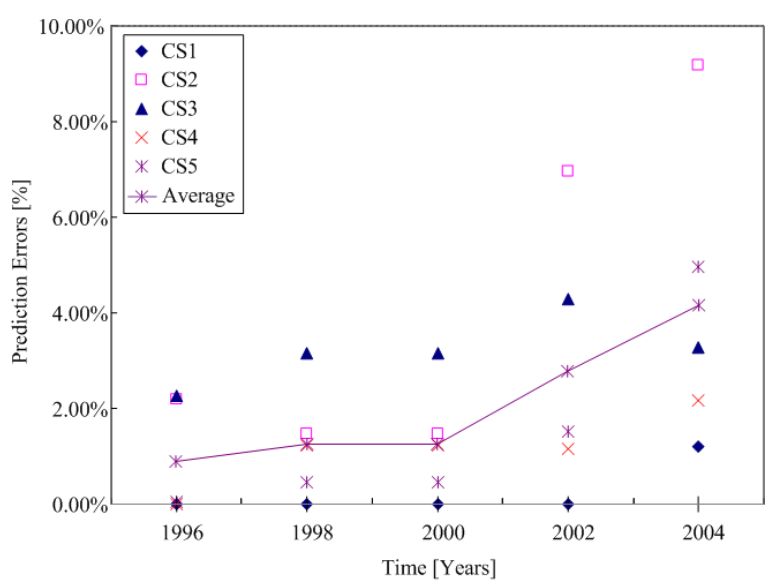

Figure 5 Prediction errors from 1996 to 2004

\section{DISCUSSION AND CONCLUSION}

In this study, the TDNN, originally designed for speech recognition, is introduced as a time-series technique to overcome limitations of existing bridge deterioration models. The primary improvement of the TDNN using BPM-generated historical condition ratings is that it can consider proportion in each CSs which cannot be considered by the existing deterministic and stochastic models.

Backward prediction results (years 1968 - 1994) are used as TDNN inputs. These input data are converted to time sequences for time series prediction in the TDNN. The proposed TDNN provides only onestep-ahead prediction at a time (one cycle). The result of the first one-step-ahead prediction (year 1996) is added on to the original TDNN input (years 1968 - 1994). Iterations of the above-mentioned process are carried out until year 2024 for a given bridge element.

In this feasibility study, the results of TDNN predictions (years 1996 - 2024) are compared with the known BMS condition ratings (year from 1996 to 2004). The annual average prediction errors are less than $\pm 10 \%$, which are acceptable. Therefore, the forecasted condition ratings (1996-2024) by the TDNN can be considered as reliable prediction outcomes, particularly for element-level condition rating.

The TDNN using BPM-generated historical condition ratings is believed to be a reliable deterioration model for satisfactory prediction of future bridge condition ratings. However, more case studies are desirable for predicting long-term bridge deterioration. Different bridge elements in different environment should be examined to confirm the prediction performance. It should also be noted that this technique can only be used for 'no maintenance' activities. To take into account the maintenance effect, Case-Based Reasoning (CBR) technique should be employed to assist prediction of optimal maintenance timing.

\section{ACKNOWLEDGEMENT}

The authors acknowledge the financial support provided by the Australian Research Council through an ARC Linkage Project (LP0883807). The authors also wish to thank the industry partners Queensland Department of Transport and Main Roads and Gold Coast City Council for their financial and in-kind support.

\section{REFERENCES}

Box, G. and Jenkins, G. (1970). “Time series analysis: Forecasting and control.” Holden-Day, San Francisco.

Bowerman, B. L., and O'connell, R. T. (1993). "Forecasting and Time Series: An Applied Approach." Duxbury Press, Belmont, California.

Czepiel, E. (1995). "Bridge Management Systems: Literature Review and Search." Northwestern University BIRL Industrial Research Laboratory.

Feng, C. X. J., Yu, Z. G., and Kusiak, A. (2006). "Selection and validation of predictive regression and neural network models based on designed experiments." Journal of IIE Transactions, 38, 13-23.

Geisser, S. (1993). "Predictive Inference: An Introduction." Chapman \& Hall, New York.

Hecht-Nielsen, R. (1990). "Neurocomputing." AddisonWesley, University of California, San Diego.

Khashei, M., Bijari, M., and Ardali, G. A. R. (2009). "Improvement of Auto-Regressive Integrated Moving Average models using Fuzzy logic and Artificial Neural Networks (ANNs)." Journal of Neurocomputing, 72, 956-967.

Kohzadi, N., Boyd, M. S., Kermanshahi, B., and Kaastra, I. (1996). "A comparison of artificial neural network and time series models for forecasting commodity prices." Journal of Neurocomputing, 10, 169-181.

Lee, J. H., Sanmugarasa, K., Loo, Y. C., and Blumenstein, M. (2008). "Improving the Reliability of a Bridge Management System (BMS) using an ANN-based Backward Prediction Model (BPM)." Journal of Automation in Construction, 17(6), 758-772.

Morcous, G., Rivard, H., and Hanna, A. M. (2002). "Modeling bridge deterioration using case-based reasoning." Journal of Infrastructure System, 8(3), 86-95.

Tan, Y., and Cauwenberghe, A. V. (1999). "Neural-networkbased d-step-ahead predictors for nonlinear systems with time delay." Journal of Aritificial Intelligence, 12, 21-35.

Waibel, A., Hanazawam, T., Hinton, G., Shikano, K., and Lang, K. J. (1989). "Phoneme recognition using time-delay neural networks." Journal of Transaction on Acoustic, Speech and Signal Processing, 37, 86-95.

Wang, X. K., Lu, W. Z., Cao, S. Y., and Fang, D. (2007). "Using Time-Delay Neural Network Combined with Genetic Algorithms to Predict Runoff Level of Linshan Watershed, Schuan, China." Journal of Hydrologic Engineering, 231236.

Zhong, M., Sharma, S., and Lingras, P. (2005). "Short-Term Traffic Prediction on Different Types of Roads with Genetically Designed Regression and Time Delay Neural Network Models." Journal of Computing in Civil Engineering 19(1), 94-103.

Zhu, J., Zurcher, J., Rao, M., and Meng, M. Q.-H. (1998). "An on-line wastewater quality prediction system based on a time-delay neural network." Journal of Aritificial Intelligence, 11, 747-758. 